\title{
ARTIFICIAL INTELLIGENCE POWERS THE DIGITAL TRANSFORMATION FOR ORGANIZATIONS
}

\author{
Lavinia-Andreea GRUIA \\ Faculty of Economics and Business Administration \\ West University of Timisoara, Romania \\ gruialavinia23@gmail.com \\ Nicolae BIBU \\ Faculty of Economics and Business Administration \\ West University of Timisoara, Romania \\ nicolae.bibu@e-uvt.ro \\ Alexandru ROJA \\ Faculty of Economics and Business Administration \\ West University of Timisoara, Romania \\ alexandru.roja@e-uvt.ro
}

\begin{abstract}
The aim of this paper is to show how organizations will use artificial intelligence (AI) to drive digital transformation. In the age of AI, organizations will have to evolve and to innovate their products and services. In this new age, organizations must learn to innovate and digitize the core of their business by making AI become the center of their digital transformation journey. Also will take a closer look at the artificial intelligence performance component this will show that for an efficient AI implementation the organization data set is very important. In this new age, data has become the new "oil" the higher the data quality an organization has the better the algorithms trained on this data will become, and in time the more efficient the AI system will become. Because implementing an AI system is not an easy task organizations must define a problem to solve and establish a strong team that will implement, deploy and monitor the artificial intelligence system.
\end{abstract}

Keywords: artificial intelligence, digital transformation, digital transformation strategy JEL classification: L86, M15, O30

DOI: $10.24818 / \mathrm{ie} 2020.02 .07$

\section{Introduction}

The world we live in is changing drastically due to the power of digitalization and innovation. One of the main impacts of this change is driven by artificial intelligence (AI) and by how organizations are using this new tool for innovation. Organizations must understand that implementing a digital transformation strategy can be a very complex and often includes the activities of thousands of people, sophisticated technologies, capital investments, and millions of lines of code that make up the operational systems and processes that enable the organization to achieve its goals [1].To better understand the disturbance, we have to understand what artificial intelligence is and which are its main business applications.

\section{Artificial intelligence}

AI represents the technology of smart machines that combine instruments and technologies with the ability to execute, learn from past experience and adapt in time [2] to find solutions. These results in three types of evaluation in AI: description, prediction, and prescription that 
can be used in combination or separated [3]. Also, AI comes in two forms: first, "strong AI" also known as Artificial General Intelligence (AGI) and this is when the machine understands what is happening, and, second,"weak AI" which is when the machine is executing simple tasks like predicting customer behaviour[3]. While for an AI system is easy to work through large data quantities using mathematical rules that is very hard for humans to process. Similarly, tasks like recognizing the meaning of objects which is very easy for humans can be very hard for an AI system to do because this is not based on mathematical rules. For example, if we train an AI model on a large data set that contains images of dogs and cats the system will easily recognize a picture of a dog or a cat. But if we show this model an image with a cat dressed as a dog it will tell us that it is a dog because it will not be able to understand that it is a dressed cat.

For a better understanding of how AI works we will describe the performance components of $\mathrm{AI}$ and their structure. The first performance component of artificial intelligence is the neural network defined as the connections between neurons that are performing a certain function. It is a concept that comes from neuroscience. Computer scientists are trying to recreate neural networks as systems whose structures are oriented towards imitating the human brain [3]. In AI such a network is composed of an input layer that receives data, multiple hidden layers that receive the data from the input layer and an output layer that generates the result of the artificial intelligence system. The second performance component is machine learning (ML) that is using calculus methods to improve performances and make predictions [1]. ML is using algorithms called self-adaptive algorithms because they are able to learn and improve themselves independently without the human programmers having to intervene. In the development of these algorithms, large quantities of data are used as training data. Also, different types of learning such as: Supervised Learning, Unsupervised Learning, and Reinforcement Learning are being used. Some of the applications of machine learning used in business are predictive maintenance, recruiting employees, customer experience and customer service. The first type of learning, "supervised learning" refers to the AI system that knows the correct answer but must adapt to the existing data to be able to give a correct answer. This type of learning uses large quantities of labelled training data as input and uses methods like linear regression and decision tree which are able to explain the price of a car model by their characteristics [3]. The second type of learning is" unsupervised learning" that is the process in which the AI system receives large quantities of unlabeled data set and is able to recognize similarities and patterns. Humans are not able to see these patterns in advance but this method lies outside of what is "humanly imaginable" [3]. These algorithms are using methods like "K-means clustering" to identify data groups that have similar characteristics also called pattern recognition [3]. The third type of learning is" reinforcement learning. It refers to the process that has no solution at the beginning of the learning and the AI system must iterate through trial and error to discover optimal solutions [3]. This process involves rewarding the system every time it makes a good decision and punish the system every time it makes a bad decision. This type of artificial intelligence can be applied to path finding, for example, robots in a warehouse must find the shortest path to get a product, every time the robot uses the shortest path will get (1) point award for every good step taken, but if the robot takes a longer path it will get a (-1) point award for every wrong step taken and the robot will perceive this as punishment.

The third component of AI is "deep learning" that is represented by specially designed neural networks and is a subset of machine learning [3]. Deep learning is able to deliver accurate results compared to traditional machine learning because it can process a wide range of data with the help of special optimization methods. This helps computers learn from self-made experience by bringing new input data. Consequently, this process allows the machine to gradually learn to assemble complex concepts from simple elements [3]. Give the above we 
can reformulate artificial intelligence such as "the core of artificial intelligence consists of independently processing large amounts of data, recognizing patterns and leading to descriptions, predictions, prescriptions or even autonomous decisions based on them" [3].

After taking a look at AI and its core elements we are going to analyse what strategies businesses are using to achieve digital transformation by making AI to become the core of their organization.

\section{Digital transformation strategy for AI implementation}

With the development of new technologies, businesses need to benefit from the advantages of digital transformation by implementing digital technologies on a large scale that will improve their operations, products and customer relationship. From predicting product demand, warehouse management to driving cars we are witnessing AI replacing traditional activities done by humans. As digital and analogue worlds are becoming one, we are entering a new age that is redefining how every organization in the economy needs to act to create, capture and deliver value [1]. We consider that AI-powered digital transformation provides new opportunities for any organization.

In order to implement a digital transformation strategy, organizations must define how they can create value, why customers are choosing to buy their products or services, which are their value proposition or customers promise, and also focus on capturing the value that it creates [1]. For example, Google gives services for free and captures value from advertisements across its products. In our opinion, this can be done because in digital business the value creation and the value capturing can be separated easily and can come from different stakeholders. To implement a digital transformation strategy for AI, we propose that business should execute the following steps: first, to identify the problem to solve, second, to create a strong team that will drive the digital transformation, third, to select the appropriate tools and platforms, fourth, to develop the artificial intelligence model, fifth, to deploy and to monitor the artificial intelligence model [4]. Also, companies that are implementing digital transformation and starting on the AI journey should define a clear business case and should put together pilot programs and develop by using experimental processes. Iansiti and Lakhani (2020) recommend for organizations that are implementing digital transformation to define an operating model that achieves value at scale, create sufficient scope and must be able to engage in sufficient learning. The scale of the operating model is defined by delivering value at a lower cost to as many customers as possible. By defining the scope as the range of activities organizations can perform (product and services variety) the operating model enables organizations to manage multiple business units or to create conglomerates. The learning of the operating model it represents an important part because organizations must be capable to continually improve over time by increasing performance and develop new products and services. Microsoft has managed to transform from a software CD company to a cloud and AI company. Microsoft's digital transformation strategy was to return back to its origins, as CEO Satya Nadella said Microsoft is "a technology company whose mission is to empower every person and every organization on the planet to achieve more". With the help of Azure services, Office 365 and Microsoft Dynamics, Microsoft aims to become the platform for the age of AI by using AI as its core. By enabling AI capabilities, Microsoft services are shifting to a services-based consumption, meaning the more the clients use the more they pay. As in the case of Microsoft, the learning of the operating model can be called the most important part because organizations must be capable to continually improve over time by increasing performance and develop new products and services.

The former head of Google Brain, Andrew Ng, has identified the main issues an organization should focus on when implementing an AI project: achieve quick win (project should take 
between 6 and 12 months to implement, also having more than one project increases the winning chance), having a meaningful project (the project should help improve the company and create additional AI investment, the value can come from lower costs, higher revenues, new business extensions, and mitigating risks), a project with an industry-specific focus ( a successful project will be a factor in boosting buy-in), and lots of data (data must be high quality and not be limited only to the data options the company has)[4].By adopting AI and reinventing the core of the organization the result will be the "AI factory" having the following characteristics: industrializing the data gathering, the analytics and the decision making process, and enabling the creation of a virtuous cycle between more user engagement, more data collection, better algorithms design, better predictions, and, finally, improvement of the services provided by the "AI factory" [1], as presented in Figure 2.

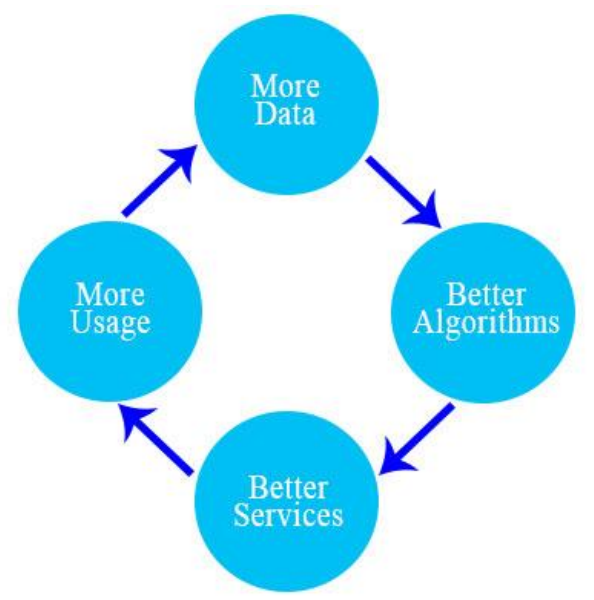

Figure 2. The AI factory's virtuous cycle[1]

These improved algorithms using the feed in data will improve over time the predictions and the accuracy of driving actions and decisions either by informing a human, or by enabling an automated response. One of the best examples for this process is represented by search engines like Google. Every time a user types a few letters the algorithm is able to predict the full search word based on the user's past actions and searched terms. Therefore, the more we search using Google, the better its predictions will become. That happens because every click is captured as a data point, and all the data points gathered are improving the predictions for future searches. The mass type of data generated in this way cannot be analyzed by humans. However, AI solves this problem by "bringing mass-production methods to data processing and analytics, thus forming the core of a digital operating model" [1].

\subsection{Principles for digital transformation}

Creating and implementing a digital transformation project represents a difficult and complex task. Iansiti and Lakhani [1] recommend five principles which can be used for an effective digital transformation. The first principle is that organizations must develop clarity of the goal and commitment to be able to deliver their business strategy. The transformation idea must bring unity to the organization because it requires recreating the organization's operating model and rebuilding it in a new and integrated foundation. Coordination becomes essential because data knows no functional boundaries. Refocusing the company on a foundation of analytics and AI requires a close and multifunctional collaboration between departments in order to improve results while reducing risks [1].The second principle of digital transformation is architectural clarity. An organization must define what its future operating architecture will be 
by setting technical goals like what data assets will be integrated across the range of applications. Also, organizations must understand the importance of standard policies as they deploy sophisticated AI to power their digital transformation. The third principle is to create an agile, product-focused organization because it is essential for an AI-centered operational model. This requires taking traditional process and embedding them in software and algorithms that will result in an AI-centric organization with a variation of AI-driven process that become the product of a modern, transformed, core service organization. The fourth principle is the capability foundation that refers to the challenge of organizations to build a foundation of capability in software, data science, and advanced analytics. Because it is very important to have the right developers, the organization must also hire and grow the data and analytics product managers about how to lead teams, how to identify useful cases to develop new applications. In the future, the role of data and analytics product managers will expand and may signal the emergence of a new generation of business leaders that can drive deeper analytics and software mindset across the organization and understand the impact of AI. The fifth principle is clear multidisciplinary governance. This principle requires that organizations must take into consideration the challenges of privacy and cyber security. As AI can bring benefits to organizations it can also unleash unintended consequences, and organizations should take into consideration deep thinking about their legal and ethical exposure. Organizations should not focus only on in-house governance but also engage with the ecosystem of partners, customers and communities. By taking into account that implementing an AI system inside of an organization is a difficult project with a lot of resources involved, organizations must have a look at the quality and value that the process creates.

Organizations that succeed in digital transformations will benefit from the important effects and generated value that the AI system deployment will create by improving [5]: a) insight quality (detecting patterns and phenomena that were never observed before such as: customer's behavior, anomalies inside production system), b) product quality(improving the product functionalities and usefulness), c) communication improvement(by introducing new channels for communication such as virtual assistants and personalized communication), d) opportunity and risk management( this will enable the identification of potentials like products, customers, and the prediction of risk factors), e) improving the quality of business processes ( this is a step that results from the above-mentioned improvements), and $\mathrm{f}$ ) the generation of a qualitatively new business model (by creating opportunities for new generation models)[5]. A good example of digital transformation using AI voice assistant is that of the Spanish company Telefónica for customer engagement. Tougher with Microsoft, Telefónica has developed the AURA AI system which is a real-time vocal system for advanced personalized customer experience, the system was build upon the data and systems the company already had [6].

By taking into account the effects of implementing an AI system as the core of the organization, we have also to take into account what could be the challenges and barriers to this process. Due to the fact that the implementation of digital transformation has been proved to be very challenging for many organizations, organizations must address the following barriers for designing and implementing [5]: a) the difficulty in finding and developing talents, b) the AI project is competing with other projects inside the organization; c) the AI system's safety aspects; d) the cultural barriers; e) lack of technological capabilities and expertise; f) lack of clarity of the business case for AI adoption; g) lack of leadership support; h) the risks resulted from the hasty implementation of immature AI solutions; i) the purchasing of solutions that don't require training and the risks of errors in implementation associated with it; $j$ ) the risk of impairing the key competencies of the organization as a result of assigning activities to the AI system [5]. Digital transformation powered by artificial intelligence represents the profound digitalization of every aspect of an organization. The digital transformation helps in the 
www.conferenceie.ase.ro

development of new competencies for innovation that lead to new benefits and opportunities for every organization on the path of transformation. Westerman affirms that for successful digital transformation organizations need technological leadership, strong relationship between the IT department and business and the right digital competencies [7].

\section{Conclusions}

As digital transformation is happening across entire industries, economy, and society, we can conclude that digital technologies and AI are enabling an increasing variety of usage. Everything is accelerated by advances in software and technologies. To implement artificial intelligence projects organizations must define a clear business case, define a problem to solve and create a strong team that will not just deliver the project but also continues to innovate and integrate different processes of business inside the project. Also, we must take into account the fact that even if artificial general intelligence (AGI) seems far into the future, the outcome is that in the end, AI will become better than humans at the operational tasks and entire systems will be automated or will become digital systems. This will result in the evolution of jobs while other jobs will disappear, as the business will continue to automate their processes. The companies that are acting to implement now an AI system based on the new principles and best practice will be the winners being more competitive.

\section{References}

[1] M. Iansiti and K. R. Lakhani, Competing in the age of AI: Strategy and leadership when algorithms and networks run the world. [On-line]. Harvard Business Review Press, ISBN 978-1633697621,

Available: https://www.amazon.com/gp/product/1633697622/ref=dbs_a_def_rwt_bibl_vppi_i0, 2020

[2] R. Akerkar, Artificial Intelligence for Business. [On-line]. Springer International Publishing, ISBN 978-3-319-97435-47. Available: https://www.springer.com/gp/book/9783319974354, 2019

[3] R. T. Kreutzer, M. Sirrenberg, Understanding Artificial Intelligence: Fundamentals, use cases and methods for a corporate AI journey. [On-line]. Springer International Publishing, ISBN 978-3-030-25270-0. Available: https://www.springer.com/gp/book/9783030252700i0, 2020

[4] T. Taulli, Artificial Intelligence Basics: A Non-Technical Introduction. [On-line].Apress, Berkeley, CA, ISBN 978-1-4842-5028-0. Available: https://link.springer.com/book/10.1007\%2F978-1-4842-5028-0, 2019

[5] A. Wodecki, Artificial Intelligence in value creation: Improving competitive advantage. [On-line]. Palgrave Macmillan, ISBN 978-3-319-91596-8. Available: https://link.springer.com/book/10.1007\%2F978-3-319-91596-8, 2019

[6] Telefónica and Microsoft establish strategic partnership to design the telco of the future: https://www.telefonica.com/en/web/press-office/-/telefonica-and-microsoft-establishstrategic-partnership-to-design-the-telco-of-the-future, 2019.

[7] G. Westerman, D. Donn, A. McAfee (October 14, 2014), Leading digital. Turning technology into business transformation. [On-line]. Harvard Business Review Press, ISBN 978-1625272478.

Available: https://www.amazon.com/gp/product/1625272472/ref=dbs_a_def_rwt_bibl_vppi_i0 [October 14, 2014] 